\title{
Preface to Special Issue on Zeolite Materials and Catalysis
}

Zeolites are crystalline microporous materials with regular channels and cages of molecular size. Due to their unique structures and acidic properties, they have been widely used in the petrochemical and chemical industries since the late 1950s. Zeolites have been used as catalysts, for instance, in fluid catalytic cracking and hydrocracking.

Faujasites were the first molecular sieves applied for catalytic purposes. Today, FAU is still the most important zeolite structure type used in catalytic transformations. Industrially important zeolites are produced synthetically. Although most industrial catalysts are drawn from the FAU, MFI, MOR, FER and BEA structure types, there are also worldwide attempts to synthesize new structure types to improve the understanding of how the zeolite structure, composition, and physical properties affect the catalytic transformation. As of now, 225 types of zeolite topology have been identified by the Structure Commission of the International Zeolite Association (IZA-SC), including over 40 naturally occurring zeolite frameworks.

This special issue is an update of the state-of-art of "Zeolite Materials and Catalysis" which covers the synthesis and characterization of zeolites and their catalytic reactions. One review, 2 communications, and 13 research articles are included in this special issue. Review paper is focused on recent advance in solid-state NMR characterization of zeolites (Feng Deng).
Communications cover the solvent-free synthesis of SAPO-5 zeolite with plate-like morphology in the presence of surfactants (Feng-Shou Xiao, Xiangju Meng) and the incorporation of cerium atoms into Al-free beta zeolite framework for catalytic application (Naijia Guan, Weili Dai). Research articles are focused on utilization of ZSM-5 zeolites with different silica/alumina ratios as FCC catalyst additives for residue cracking (Jihong Yu), synthesis and characterization of Eni carbon silicates prepared in the Si/Ga system (Giuseppe Bellussi, Michela Bellettato), One-pot synthesis of 5-Hydroxymethyl furfural from glucose using bifunctional [Sn,Al]-Beta catalysts (Peng $\mathrm{Wu}$ ), activation of Mo/HZSM-5 for methane aromatization (Emiel J. M. Hensen), ordered mesoporous silicalite-1 zeolite assembled from colloidal nanocrystalline precursors (Weiguo Song), heterogeneous oxidation of cyclohexanone catalyzed by titanium silicate zeolite TS-1: combined experimental and DFT studies (Xiaodong Zou), ionothermal synthesis of zeolitic imidazolate frameworks and the synthesis dissolution-crystallization mechanism (Zhijian Tian), one-pot synthesis of hierarchically structured ZSM-5 zeolites using single micropore-template (Jianlin Shi, Zile Hua), location and orientation of the $\mathrm{N}, \mathrm{N}$-diisopropyl ethylamine template in the AEI framework by X-ray synchrotron diffraction and molecular modelling (Sankar Gopinathan), influence of zinc species on the reaction process

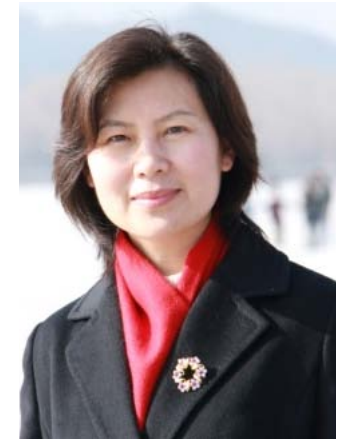

Jihong Yu (Guest Editor) State key Laboratory of Inorganic Synthesis and Preparative Chemistry, College of Chemistry, Jilin University jihong@jlu.edu.cn

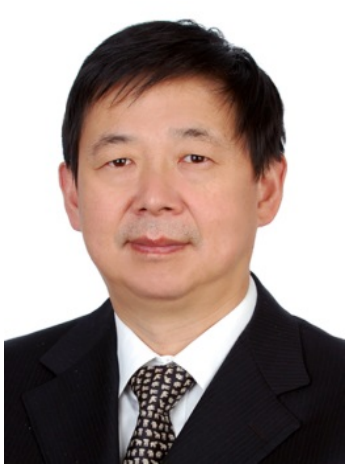

Feng-Shou Xiao (Guest Editor) Department of Chemistry, Zhejiang University fsxiao@zju.edu.cn

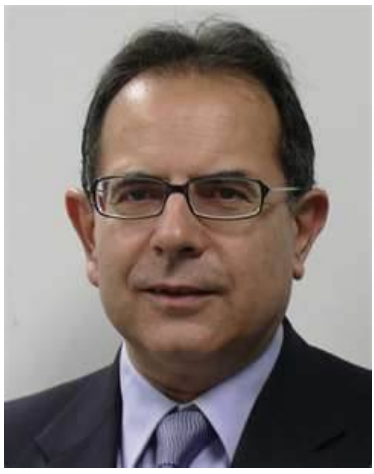

Avelino Corma (Guest Editor) Universidad Politécnica de Valencia, 46022 Valencia, Spain acorma@itq.upv.es 
of ethylene aromatization (Jianguo Wang, Weibin Fan), the influence of $\mathrm{Al}^{3+}$ on the enrichment of polymorph $\mathrm{A}$ in the crystallization of zeolite beta (Wenfu Yan), Co-ETS-10 and Co-AM-6 as active catalysts for facile oxidation of styrene into styrene oxide and benzaldehyde with molecular oxygen (Kyung Byung Yoon), and post-synthesis of hierarchically structured Ti-BEA zeolites and their epoxidation catalytic performance (Jianlin Shi, Zile Hua). The high quality of the papers written by international well-known research groups in this highly exciting research area will render this special issue a good reference for future research activity.

We express our sincere thanks to all the authors. 